\title{
Radiation-Induced Heart Disease: A Clinical Update
}

\author{
Syed Wamique Yusuf, Shehzad Sami, and Iyad N. Daher \\ Department of Cardiology, University of Texas MD Anderson Cancer Center, Unit 1451, Houston, TX 77030, USA \\ Correspondence should be addressed to Syed Wamique Yusuf, syusuf@mdanderson.org
}

Received 14 October 2010; Accepted 15 December 2010

Academic Editor: Jean-Bernard Durand

Copyright () 2011 Syed Wamique Yusuf et al. This is an open access article distributed under the Creative Commons Attribution License, which permits unrestricted use, distribution, and reproduction in any medium, provided the original work is properly cited.

Cardiovascular diseases and cancer are the two leading causes of morbidity and mortality worldwide. Improvement in cancer therapy has led to increasing number of cancer survivors, some of whom may suffer from adverse cardiovascular effects of radiation therapy. Longterm followup is essential, as the cardiac complication may manifest years after completion of radiation therapy. In this paper, we have discussed the cardiovascular effects of radiation therapy.

\section{Introduction}

Cardiovascular disease and cancer are the two leading causes of morbidity and mortality worldwide [1]. Over the last half century, radiation therapy (RT) has evolved to become one of the cornerstones of treatment for various types of cancers. It is estimated that more than $50 \%$ of patients with cancer are treated with radiotherapy. Along with the development of novel chemotherapeutic agents, radiation therapy (RT) has revolutionized the prognosis of patients with various cancers. Many childhood and adolescent years cancers are now successfully treated and these patients go on to live an active and normal adult life, as evident by an increasing number of cancer survivors [2]. Late cardiovascular effects are seen often in cancer survivors. Amongst Hodgkin lymphoma (HL) patients who have received radiation, cardiovascular disease (CVD) is of the most common causes of death. Studies have shown that these patients have an increased risk for Coronary Artery Disease (CAD), valvular heart disease, congestive heart failure $(\mathrm{CHF})$, pericardial disease and sudden death. The risk is particularly high in patients treated before the age of 40 years [3-6].

The reported relative risk of death from a fatal myocardial infarction in patients treated with mediastinal RT is increased from 1.5 to 3.0 times that of unirradiated patients $[7,8]$. In young patients undergoing mediastinal irradiation, myocardial ischemia and CAD is very prevalent [9]. A meta-analysis of eight randomized trials found a
$62 \%$ increase in cardiac deaths among women who were treated with radiation therapy [10]. Even at lower radiation doses, there appears to be of excess risk of cardiovascular disease as shown in the Japanese atomic bomb survivors [11]. Due to improvement in radiation technique, the risk of cardiovascular complications in relation to radiation may have declined over time, but even in patients treated as lately as between 1979 and 1986 the risk congestive heart failure and valvular dysfunction remained increased [12]. Radiation damage to the heart can involve the pericardium, myocardium, valves, and coronary vessels with pericardium being most frequently involved $[13,14]$. Radiation damages the vascular endothelium, and hence radiation-induced vascular injury occurs in the field of radiation exposure. Damage to the capillary vessels manifests as telangiectasia, whereas thrombotic, inflammatory, and fibrogenic complications in larger vessels can result in peripheral, coronary and carotid artery disease. Table 1 lists the possible radiation induced cardiovascular complications.

Figure 1 illustrates the case of a patient who had multiple cardiovascular complications in relation to radiation therapy. Patient had Hodgkin's disease and completed radiation therapy (total of $40 \mathrm{~Gy}$ ) to the mediastinum and neck in 1984. In 2005, a permanent pacemaker was inserted for complete heart block. In March 2007, he underwent pericardiocentesis, followed by a pericardial window. Subsequently he underwent pericardial stripping for pericardial constriction in April 2007. He also developed CAD and was 
TABLe 1: Spectrum of radiation induced heart disease.

(1) Radiation-induced atherosclerosis
(a) Symptomatic
(b) Asymptomatic

(2) Pericardial disease
(a) Acute pericarditis
(b) Delayed pericarditis
(c) Pericardial effusion
(d) Constrictive pericarditis

(3) Myocardial and Endocardial disease
(a) Pancarditis
(b) Cardiomyopathy

(4) Valvular disease

(5) Conduction disturbances
(a) $R B B B$
(b) Atrioventricular nodal block

found to be $50 \%$ disease of the left anterior descending artery. His other radiation-included complications included hypothyroidism and restrictive lung disease.

\section{Possible Mechanism of Radiation-Induced Vascular Disease}

The exact primary mechanisms by which radiation-induces or promotes atherosclerosis have not been identified. A number of acute effects, including endothelial damage, lipid and inflammatory cell infiltration, and lysosomal activation, have been described $[15,16]$. Primary basic mechanism behind radiation induced vascular damage appear to be endothelial dysfunction [17]. However certain risk factors like smoking and hyperlipidemia appears to act as accelerating agents. Radiation itself can cause fibrotic changes in the coronary arteries [14], but cholesterol feeding of the animals markedly increased the degree of atherosclerosis in the rabbits [18]. These findings suggest that the combined effect of irradiation and some other risk factor is necessary to produce significant radiation-induced atherosclerosis [18].

Though previously initiation of atherosclerosis was attributed mainly to lipid accumulation within the arterial walls, it is now widely accepted that inflammation plays a vital role in the initiation and progression of the disease [19, 20]. In animal model irradiation accelerates the development of macrophage-rich, inflammatory atherosclerotic lesions prone to intraplaque hemorrhage [21] and enhances the atherogenic effects of a high-fat diet [22]. It is postulated that radiation promotes short-lived changes in oxidative stress conditions (O2 concentrations) in the artery wall and that atherogenic lipoproteins, which are elevated in response to the high-fat diet, must be available during this period to participate in the initiation of the disease process [22]. Another mechanism of radiation-induced endothelial dysfunction is via production of reactive oxygen species [23]. Significant increases in superoxide and peroxides were

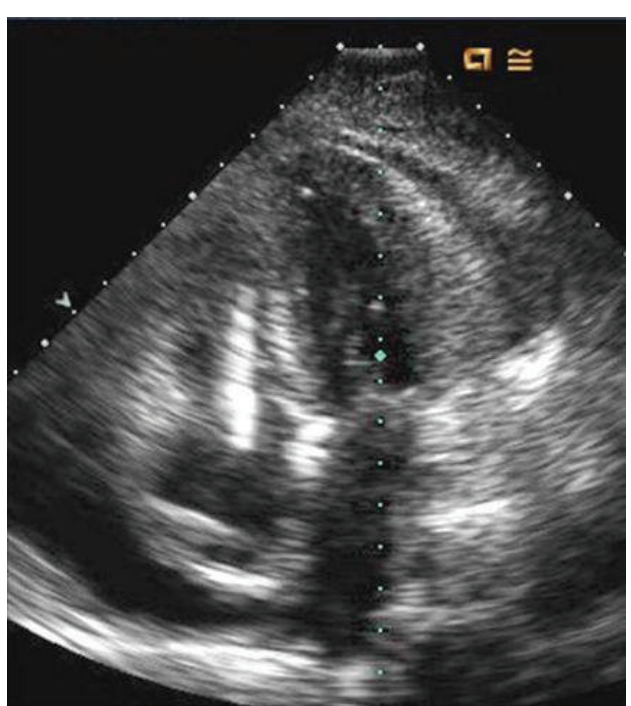

Figure 1: Echocardiogram image (apical 4-chamber view) shows thickened pericardium, small pericardial effusion, and a pacemaker lead in the right ventricle.

observed in the microvessels of rats exposed to radiation [23].

A proposed mechanism of inflammatory vascular damage due to radiation in humans is also via oxidative stress and activation of nuclear factor-kappa B ( NF-kB) [24].

Certain cytokines and growth factors, such as TGF-beta1 and IL-1 beta, may stimulate radiation-induced endothelial proliferation, fibroblast proliferation, collagen deposition, and fibrosis leading to advanced lesions of atherosclerosis [25].

Indirect association of inflammation with radiationinduced vascular damage comes from studies showing elevated levels of the proinflammatory cytokines IL-6, CRP, TNF- $\alpha$, and INF- $\gamma$ and also increased levels of the antiinflammatory cytokine IL-10, in the Japanese atomic bomb survivors $[26,27]$. There was also dose-related elevation in erythrocyte sedimentation rate and in levels of IgG, IgA and total immunoglobulins in this cohort, all markers of systemic inflammation [27]. In experimental atherosclerosis model it has been shown that following multiple small radiation doses the chemoattractant (MCP-1) concentration increases proportionally to cumulative dose; this increase in MCP-1 is largely driven by radiation-induced monocyte death [28]. In an animal model, following a single dose of radiation to the heart, from 3 months onwards changes in coronary arteries of the irradiated hearts included endothelial cell loss, a loss of smooth muscle cells, and fibrosis in media and adventitia [29].

Endothelial cell injury markers secreted after irradiation includes thrombomodulin [30]. Following radiation, the endothelial cell neutrophil chemotactic activity is increased, with greater adherence of polymorphonuclear leucocytes to irradiated endothelial cells [31]. In women with breast cancer, it has been shown that endothelium-dependent vasodilatation was significantly impaired in the irradiated axillary arteries compared with the contra lateral, nonirradiated arteries [32]. In irradiated human cervical arteries, 
the impaired nitric oxide-mediated relaxation was associated with a lack of endothelial nitric oxide synthase expression, suggesting the importance of impaired endothelial function in irradiated human blood vessels [33]. Endothelial cell swelling, increased permeability, interstitial fibrin deposition, and development of platelet thrombi ultimately lead to fibrosis. Various local and systemic factors, implicating endothelial cell damage and inflammation, eventually lead to the development of symptomatic CAD.

\section{Radiation-Induced CAD}

Due to high prevalence of CAD, the precise incidence of radiation induced accelerated atherosclerosis is difficult to confirm. However it occurs in a number of patients without traditional risk factors for $\mathrm{CAD}[9,34]$. In these patients, conventional risk factors like CAD, diabetes mellitus, hyperlipidemia, and smoking were uncommon and insensitive indicators of coronary risk after mediastinal irradiation [9].

3.1. Pathology. Intimal proliferation of mainly fibrous tissue leads to luminal narrowing [14]. Histologically there may be some overlap and it may be difficult to distinguish between radiation-induced CAD (RICAD) and typical atherosclerotic $\mathrm{CAD}$. However media is more severely destroyed and the adventitia is markedly thickened and fibrotic in radiationinduced CAD as compared with nonradiation CAD [35]. In irradiated coronary vessels, there also appears to be loss of smooth muscle cells from the media and adventitial fibrosis [14].

3.2. Clinical Presentation. Symptoms of CAD may include chest discomfort with or without radiation to the arm, back, neck, jaw, or epigastrium, shortness of breath, weakness, diaphoresis, nausea, and lightheadedness, all with or without exertion. Women and the elderly are more likely to present with "atypical features" [36]. Clinically patients with radiation induced CAD (RICAD), most commonly, present with angina, dyspnea or heart failure [34]; however there are reports of sudden death in patients treated with radiotherapy [14]. Sudden death in these patients is thought either to diffuse fibrointimal hyperplasia of all coronary vessels or left main stenosis and ostial lesions [14, 34]. Mean time interval for the development of RICAD in relation to radiotherapy is approximately 82 months (range 59-104) [13]. Patients with RICAD generally present at younger age than general population, especially survivor of childhood and adolescence malignancies treated with mediastinal radiation [37]. Hancock et al. followed a total of 2232 consecutive Hodgkin's disease patients treated from 1960 through 1991 for an average of 9.5 years to assess the risk of death from heart disease after Hodgkin's disease therapy. The relative risk (RR) for cardiac death was found to be 3.1 (CI; 2.4-3.7) and the RR for acute myocardial infarction was the highest after irradiation before 20 years of age and decreased with increasing age at treatment $(P<$ .0001 for trend) [8]. The proximal coronary vessels, which are in the range of radiation, are mainly involved $[13,14]$.
Occasionally in patient with myocardial infarction due to previous radiation therapy, no obstructive CAD is found and provocative tests for coronary artery spasm response are normal [38]. Spontaneous recanalization of the thrombus may have occurred as this patient was treated with aspirin and heparin and coronary angiogram was done 3 days later [38].

Asymptomatic disease is also prevalent in patients with previous radiation. New perfusion defects occurred in 50\% to $63 \%$ of women 6 to 24 months after RT [39, 40]. Marks et al. demonstrate 27\%, 29\%, 38\%, and $42 \%$ incidence of myocardial perfusion abnormalities in asymptomatic patients with breast cancer at $6,12,18$, and 24 months after $\mathrm{RT}$, respectively [41]. The incidence of perfusion defects was strongly correlated with the volume of left ventricle $(\mathrm{LV})$ in the RT field occurring in $25 \%$ of patients with $1 \%$ to $5 \%$ of the LV within the tangent fields, and in 55\% of patients with more than $5 \%$ of the LV within the field [41]. The clinical significance of these perfusion defects is unknown. However, they appear to be associated with abnormalities in wall motion and episodes of chest pain [41, 42]. A nonsignificant change in ejection fraction is apparent only in patients with relatively large fractions of the LV affected by perfusion defects [41]. In patients with distal esophageal cancer, RT is associated with a high prevalence of inferior left ventricular ischemia, as detected by gated myocardial perfusion images (GMPIs) [43]. Most perfusion defects are encompassed within an isodose line $\geq 45 \mathrm{~Gy}$ in the RT plan, and most patients have mild degree of ischemia [43]. A wall motion abnormality was seen in only few patients; such finding is likely due to the presence of only mild degree of ischemia [43]. In a small group of patients with esophageal and lung carcinoma, myocardial perfusion abnormalities were frequently seen after radiation but were not predictive of future cardiac events [44]. Due to small number of patients in these series and short duration of followup [41, 42, 44], the long-term clinical significance of these mild perfusion defects is unknown.

3.3. Diagnosis and Treatment. Diagnosis and treatment of radiation-induced CAD (RICAD) is similar to CAD in general population [45]. There are no specific guidelines or significant difference in the acute initial stabilization and management of these patients. Generally the treatment of both acute and chronic RICAD would follow the same lines as management of atherosclerotic CAD either with medical therapy or revascularization, considering the patient's symptoms, cancer stage, expected survival, and comorbidities.

For patients with RICAD, both percutaneous intervention and coronary artery bypass graft (CABG) have been used [34]. Surgical intervention and CABG may pose difficulties in these patients because of mediastinal fibrosis, with high incidence of complications [34]. In addition, the use of internal mammary artery as a graft may not always be possible due to radiation disease with this vessel itself.

Figure 2 represents a CT coronary angiogram showing left main disease in a 60-year-old female who had received radiation therapy to the mediastinum. 


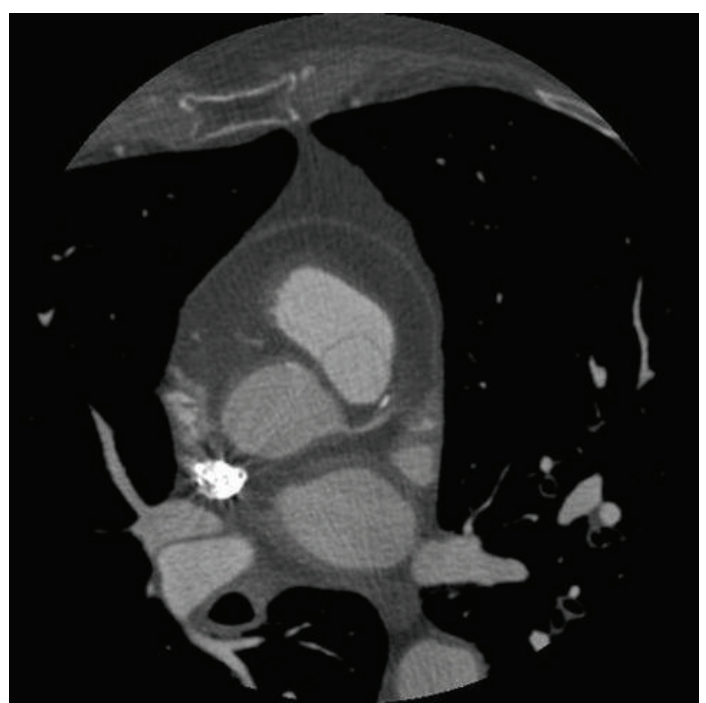

FIgURE 2: CT coronary angiogram showing left main disease in a 60-year-old female who had received radiation therapy the mediastinum.

\section{Radiation-Induced Carotid Artery Disease}

In patients with Hodgkin's disease, when compared to controls, the irradiated cases had a greater intima-media thickness in the carotid artery distribution [46]. During a 3-year followup, it was noted that despite improvement in intima-media thickens at 3 years, there was persistently reduced flow-mediated dilatation in the irradiated carotid artery, suggesting that early endothelial damage persists [47]. Radiation-induced carotid disease differs from traditional carotid disease. Radiotherapy often produces carotid lesions that are more extensive than the traditional bifurcation stenosis, often involving atypical areas such as long segments of carotid artery [48].

The treatment of radiation-induced carotid disease is similar to those in general population with both percutaneous intervention and surgery used in selective cases [49].

In patients with extensive fibrosis due to radiation, carotid stenting may be preferable.

Figure 3 represents a carotid Doppler of a 33-year-old female who had received head and neck radiation for nasopharyngeal carcinoma 18 yrs ago, showing moderate disease in the left common carotid artery. The patient also had evidence of occlusion of the left subclavian artery.

\section{Radiation-Induced Pericardial Disease}

Pericardial disease is a common manifestation of radiationinduced heart disease and is most frequently involved. In an autopsy study of radiation-induced heart disease, radiationinduced pericardial disease was noted in $70 \%$ of cases in whom pericardium was available [13].

5.1. Pathophysiology. Fibrosis is a common sequel of radiotherapy. It is a multicellular process involving various

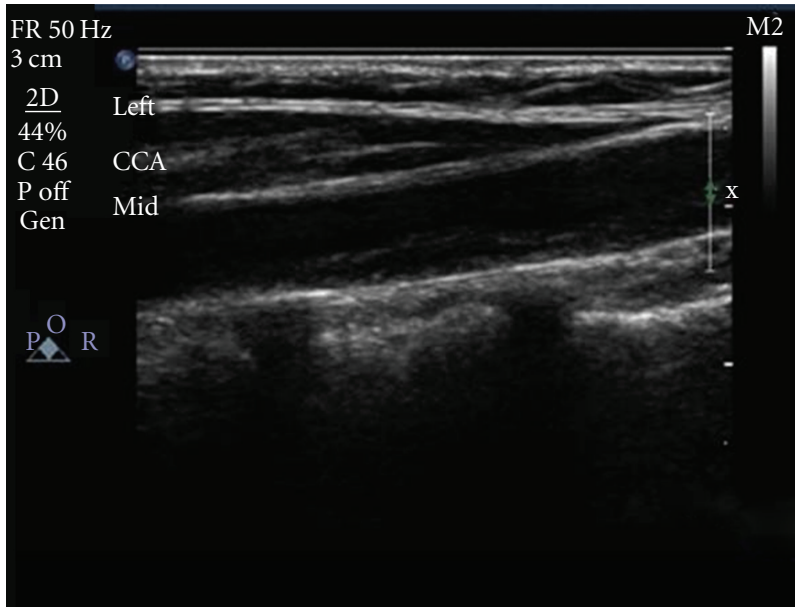

Figure 3: A carotid Doppler shows moderate disease in the left common carotid artery of a 33-year-old female who had received head and neck radiation for nasopharyngeal carcinoma 18 years ago.

interacting cell systems in the heart resulting in the fibrotic phenotype of the fibroblast/fibrocyte cell system, which usually causes fibrous thickening of the pericardium [50]. In the pericardium, parietal surfaces are more severely damaged, with dense collagen and fibrin deposition on the mesothelial surfaces [51].

5.2. Clinical Presentation, Diagnosis, and Treatment. Pericardial disease with radiotherapy can present as pericarditis, pericardial effusion with or without constrictive pericarditis [13]. Pericardial effusion with or without constriction is the most commonly observed type of pericardial disease [13].

In patients with radiation-induced pericardial disease, the interval from radiation therapy to symptom development is variable and ranges between 2 and 145 months (mean time: 58 months) [13]. Patients presenting earliest were those with effusions, whereas those with constriction all became symptomatic after 18 months [13]. The development of disease 12 years after treatment emphasizes the importance of long-term followup in these patients [13].

Pericardial disease with radiotherapy can present as the following cases.

(1) Acute pericarditis during radiation treatment: the signs and symptoms are those of nonspecific pericarditis with chest pain, fever, and sometimes nonspecific ECG abnormalities. (Figure 4 represents an ECG showing pericarditis in a 60-yearold female who presented with chest pain 7 days after starting radiation therapy for breast carcinoma). Acute pericarditis during radiation treatment generally responds to bed rest and nonsteroidal antiinflammatory drugs (NSAIDs). Treatment of primary malignancy should not be withheld because of this.

(2) Delayed pericardial disease: it can occur from months to several years after radiotherapy $[52,53]$. Patients 


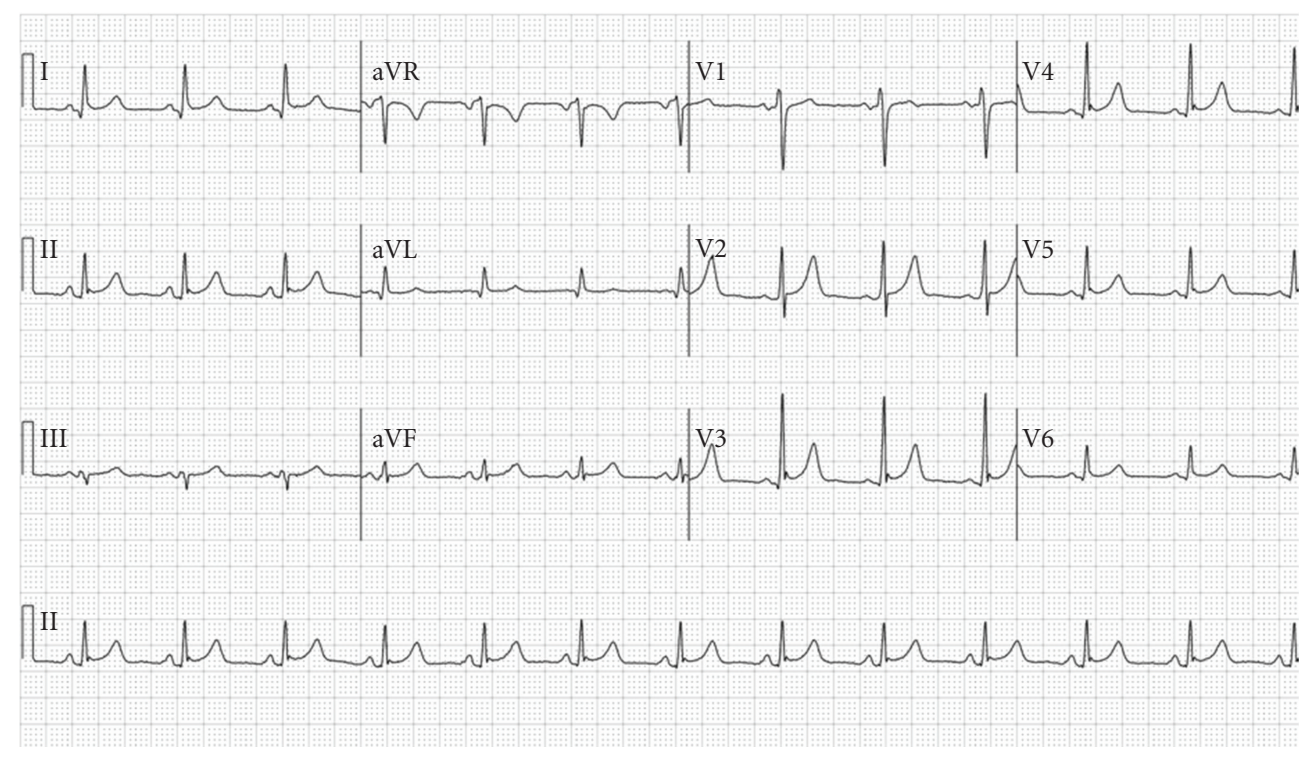

FIGURE 4: ECG showing ST elevation suggestive of pericarditis.

may present with pericardial pain, but dyspnea is the commonest presenting symptom $[52,53]$.

Delayed chronic pericardial effusion: it may present within 4-26 months and is usually detected by chest $\mathrm{X}$ ray showing an enlarging cardiac silhouette [52]. Radiographic evidence of cardiomegaly usually precedes the development of dyspnea and other signs of tamponade [52]. Some patients may present with tamponade. When delayed pericardial disease presents with a cardiac tamponade like clinical picture, needle pericardiocentesis is usually the initial treatment of choice. Recurrent, large, and hemodynamically significant pericardial effusions require surgical pericardiectomy, if no contraindications exist [52].

(3) Pancarditis: the most severe form of radiationinduced pericardial disease is pancarditis. In addition to pericardial fibrosis, there is myocardial fibrosis with or without endocardial fibrosis or fibroelastosis. Clinical picture is that of intractable congestive heart failure. When radiation-induced pancarditis presents as acute decompensated heart failure, besides early risk stratification, patients should be treated with loop diuretics, nitroglycerin, ACE inhibitors, vasodilators, inotropic agents, and supportive device as indicated.

(4) Constrictive Pericarditis: mediastinal radiation is a common cause of constrictive Pericarditis [54]. The majority of the patients present many years after radiation therapy with congestive heart failure [54]. Congestive heart failure is treated with conventional medications like diuretics, beta blockers and ACE inhibitors. The definite treatment is pericardiectomy, but radiation therapy is associated with poor survival in these patients, and hence pericardiectomy may not be curative for patients with radiation-induced constrictive pericarditis [54].

(5) In clinical practice some asymptomatic patients may have minimal to small pericardial effusion on routine echocardiogram. Except for followup, no active intervention is needed in these cases.

\section{Radiation-Induced Cardiomyopathy}

A large number of patients with previous radiation therapy develop cardiomyopathy. In a retrospective cohort study involving 14,358 five-year survivors of various cancers diagnosed and treated under the age of 21 between 1970 and 1986, Mulrooney et al. found that survivors of cancer were significantly more likely than siblings to report congestive heart failure (hazard ratio (HR) 5.9, 95\% confidence interval 3.4 to $9.6 ; P<.001)$. Exposure to $250 \mathrm{mg} / \mathrm{m} 2$ or more of anthracyclines and radiation dose of more than $1200 \mathrm{cGy}$ to the heart increased the relative hazard of congestive heart failure by two to sixfold as compared to nonirradiated survivors [55].

Overall, heart failure is a rare sequel of mediastinal radiation therapy and mostly associated with a restrictive hemodynamic pattern in the absence of a history of treatment with an anthracycline [56]. At a microscopic level collagen not only increases as a whole but the proportion of type I collagen increases proportionally to type 111 . This marked alteration in collagen synthesis may contribute to impaired diastolic distensibility of the ventricles seen in this group of patients [57]. Most patients with myocardial involvement have interstitial fibrosis [13]. The most common pattern of fibrosis is pericellular and perivascular, with replacement fibrosis seen infrequently [13]. The loss of myocardium results in renin-angiotensin-aldosterone (RAA) and adrenergic system-driven myocardial remodeling, which is progressive and results in end-stage symptoms. 
Multiple case series have reported myocardial perfusion defects in patients receiving mediastinal radiation therapy $[39,58-60]$. The significance of these perfusion defects is unclear. However, they likely represent microvascular damage to the myocardium, which over time may lead to myocardial fibrosis and diastolic dysfunction [61]. Even if acute myocardial damage is only moderate, the process of myocardial remodeling can lead to progressive myocardial dysfunction over years and eventually induce myocardial dysfunction and heart failure. Treatment with ACE inhibitors, angiotensin receptor blockers, aldosterone antagonists, and beta blockers is usually recommended as per guidelines for treatment of heart failure in general population [62]. In animal model, captopril (an ACE inhibitor) ameliorated many of the structural changes but failed to influence or prevent the late functional deterioration after irradiation of the heart [63].

\section{Radiation-Induced Valvular Heart Disease}

Valvular disease is frequently seen in patients with radiationinduced heart disease. In one postmortem series, the incidence was high with $81 \%$ of patients showing evidence of valvular damage [14]. The aortic and mitral valves are more commonly involved than the tricuspid and pulmonary valves $[14,64]$. The reason for preponderance of left-sided lesion and the rarity of pulmonary valve involvement despite its anterior position is not known. This may be related to higher pressure across left-side valves. Only a minority of patients with radiation-associated valvular disease (RAVD) have clinically moderate or severe dysfunction [14]. Vast majority $(71 \%)$ of patients with RAVD exhibit no symptoms of valvular dysfunction [65]. The mean time from radiation to the onset of symptoms in RAVD is approximately 98 months (range 18-252) [13]. In an individual patient exposed to radiation, it is difficult to assess the time period for conversion of asymptomatic to symptomatic valvular heart disease, as radiation-associated valvular heart disease represents a continuum progressing from asymptomatic valvular thickening to symptomatic valvular dysfunction. However, it has been noted that diagnosis of asymptomatic RAVD occurred at a mean of 11.5 years after irradiation compared with 16.5 years for symptomatic patients [65]. Thus, 5 years appear to be the interval required for progression from asymptomatic to symptomatic RAVD.

The valve leaflets are fibrotic with focal dystrophic calcification and marked thickening. In the pathological findings, certain rheumatic endocarditis changes such as endocardial reduplication and vascularization are not found [64]. The cusps or leaflets of the valves thicken fibrose and on occasion partially calcify $[66,67]$. The treatment of valvular disease in cancer patients and those related to radiotherapy is the same as in general population. The issue of endocarditis prophylaxis should be individualized.

Figure 5 represents Doppler echocardiogram of the aortic valve in a 46-year-female showing moderate aortic stenosis and aortic regurgitation. The patient had received radiation therapy for Hodgkin's disease 29 years ago. Some degree of regurgitation was noted in all 4 valves.

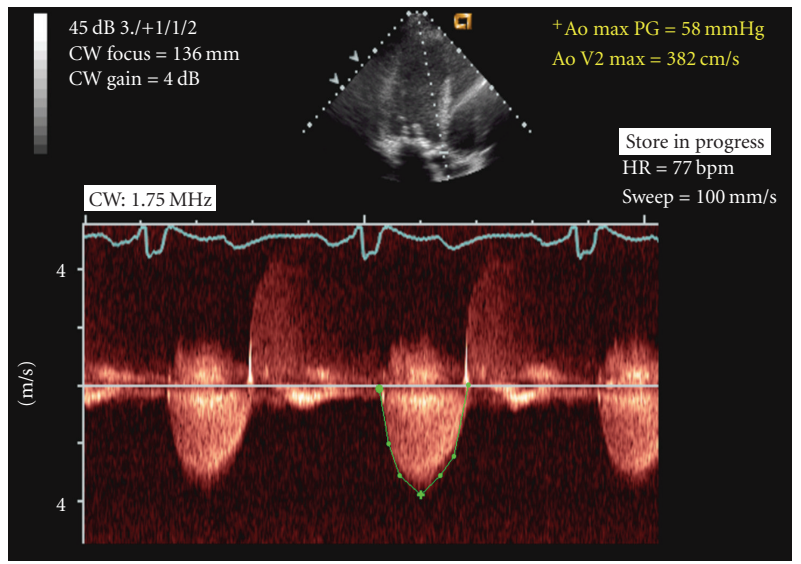

FIGURE 5: Doppler echocardiogram of the aortic valve (done in 2005) shows moderate aortic stenosis and aortic regurgitation in a 46-year-female who had received radiation therapy for Hodgkin's disease in 1976. Some degree of regurgitation was noted in all 4 valves.

\section{Radiation-Induced Conduction System Disease}

The conduction system is the least commonly involved of all the cardiac structures and its true incidence is not known. ECG changes after irradiation range from nonspecific ST$\mathrm{T}$ changes to low voltage and right bundle branch block (RBBB) [14]. RBBB is commonly associated with mediastinal irradiation because the right bundle lies close to the endocardium on the right side [68]. Injury to bundle may occur either directly from radiation or indirectly from associated myocardial fibrosis and ischemia. One of the common serious manifestations is complete atrioventricular (AV) block [69]. Dual chamber pacing has been found to be somewhat more efficacious in symptom improvement in such patients [70]. A postmortem study in a 20-yearold man with Hodgkin's disease and previous radiation who had earlier developed heart block revealed marked arteriosclerosis with fibrosis of the epicardium, myocardium, and endocardium. Examination of the conduction system revealed extensive arteriolosclerosis of the sinoatrial node and its approaches. In addition, there was marked fibrosis of the approaches to the AV node, the AV bundle, and both bundle branches [71]. Complete heart block has been reported to occur at an interval of less than 1 year from radiation to 23 years after completion of radiation therapy [72]. Hence these patients should be followed up for life.

\section{Role of Biomarkers in Patients Undergoing Radiation Therapy}

There are only few studies of biomarkers in patients undergoing radiation and have shown conflicting results. In a study of 50 women with breast cancer, no change in serum troponin was found after a total dose of 45 to $46 \mathrm{~Gy}$ [73]. In this study, however, the cardiac dosimetric data was not provided [73]. In another study of 30 patients receiving 
thoracic chemoradiation, no significant elevation in creatine kinase-myocardial band (CK-MB), troponin, or NT-pro BNP was detected with radiation therapy [74]. In one study both troponin and BNP increased significantly during the study; however, the absolute and mean values remained on a relatively low level (mean preradiation and postradiation troponin I: $0.007 \pm 0.008,0.014 \pm 0.01 \mathrm{ng} / \mathrm{mL}$; mean preradiation and post radiation BNP: $123 \pm 147,159 \pm 184 \mathrm{pg} / \mathrm{mL}$ ) [75]. Hence based on current knowledge, cardiac biomarkers are not recommended for evaluation of radiation-induced cardiotoxicity but remain a useful research tool.

\section{Prevention and Future Direction}

Subclinical cardiac damage occurs in $>50 \%$ of breast cancer survivors treated with radiation therapy [39]. Alteration in radiotherapy field or targeted radiation, with avoidance and/or shielding of the heart, remains one of the most important things in prevention of radiation-induced cardiac damage. In the absence of risk factors, the value of primary and secondary prevention in these cases is debatable. Patients with classical risk factors like hypertension, smoking, and hyperlipidemia may be at increased risk of radiation-related cardiovascular complications, and these risk factors should be treated aggressively. Younger patients should be screened, because this patient population at risk usually have a considerable life expectancy. The role of routine stress test and biomarkers like troponin and B-type natriuretic peptide (BNP) in identifying high risk patients or predicting future cardiovascular events remains to be determined in large studies.

Similarly, in the absence of risk factors, the role of prevention therapy like use of antiplatelets, ACE inhibitors, and lipid lowering agents remains unclear. Recently CT scan of the coronary artery has been identified as a useful tool in identifying RICAD in asymptomatic patients [76]. Studies of myocardial perfusion in patients with previous radiation therapy indicate that the majority have mild myocardial perfusion defects. In patients with good long-term prognosis, the finding of the ischemia due to the RT may be significant as this is a reversible and treatable stage of RICAD, and halting its progression may decrease the incidence of future cardiovascular events.

\section{Funding}

The authors received no funding.

\section{Conflict of Interests}

There is no conflict of interests.

\section{References}

[1] V. Fuster and J. Voûte, "MDGs: chronic diseases are not on the agenda," The Lancet, vol. 366, no. 9496, pp. 1512-1514, 2005.

[2] W. A. Bleyer, "The impact of childhood cancer on the United States and the world," Ca: A Cancer Journal for Clinicians, vol. 40, no. 6, pp. 355-367, 1990.
[3] B. M. P. Aleman, A. W. van den Belt-Dusebout, W. J. Klokman, M. B. Van't Veer, H. Bartelink, and F. E. van Leeuwen, "Longterm cause-specific mortality of patients treated for Hodgkin's disease," Journal of Clinical Oncology, vol. 21, no. 18, pp. 34313439, 2003.

[4] R. T. Hoppe, "Hodgkin's disease: complications of therapy and excess mortality," Annals of Oncology, vol. 8, no. 1, pp. S115S118, 1997.

[5] A. K. Ng, M. P. Bernardo, E. Weller et al., "Long-term survival and competing causes of death in patients with early-stage Hodgkin's disease treated at age 50 or younger," Journal of Clinical Oncology, vol. 20, no. 8, pp. 2101-2108, 2002.

[6] A. J. Swerdlow, C. D. Higgins, P. Smith et al., "Myocardial infarction mortality risk after treatment for hodgkin disease: a collaborative British cohort study," Journal of the National Cancer Institute, vol. 99, no. 3, pp. 206-214, 2007.

[7] J. F. Boivin, G. B. Hutchison, J. H. Lubin, and P. Mauch, "Coronary artery disease mortality in patients treated for Hodgkin's disease," Cancer, vol. 69, no. 5, pp. 1241-1247, 1992.

[8] S. L. Hancock, M. A. Tucker, and R. T. Hoppe, "Factors affecting late mortality from heart disease after treatment of Hodgkin's disease," Journal of the American Medical Association, vol. 270, no. 16, pp. 1949-1955, 1993.

[9] P. A. Heidenreich, I. Schnittger, H. W. Strauss et al., "Screening for coronary artery disease after mediastinal irradiation for Hodgkin's disease," Journal of Clinical Oncology, vol. 25, no. 1, pp. 43-49, 2007.

[10] J. Cuzick, H. Stewart, L. Rutqvist et al., "Cause-specific mortality in long-term survivors of breast cancer who participated in trials of radiotherapy," Journal of Clinical Oncology, vol. 12, no. 3, pp. 447-453, 1994.

[11] Y. Shimizu, D. A. Pierce, D. L. Preston, and K. Mabuchi, "Studies of the mortality of atomic bomb survivors. Report 12, part II. Noncancer mortality: 1950-1990," Radiation Research, vol. 152, no. 4, pp. 374-389, 1999.

[12] M. J. Hooning, A. Botma, B. M. P. Aleman et al., "Long-term risk of cardiovascular disease in 10-year survivors of breast cancer," Journal of the National Cancer Institute, vol. 99, no. 5, pp. 365-375, 2007.

[13] J. P. Veinot and W. D. Edwards, "Pathology of radiationinduced heart disease: a surgical and autopsy study of 27 cases," Human Pathology, vol. 27, no. 8, pp. 766-773, 1996.

[14] F. C. Brosius, B. F. Waller, and W. C. Roberts, "Radiation heart disease. Analysis of 16 young (aged 15 to 33 years) necropsy patients who received over 3,500 rads to the heart," American Journal of Medicine, vol. 70, no. 3, pp. 519-530, 1981.

[15] A. W. T. Konings, M. J. Hardonk, R. A. Wieringa andLamberts, and H. B. Lamberts, "Initial events in radiation induced atheromatosis I. Activation of lysosomal enzymes," Strahlentherapie, vol. 150, no. 4, pp. 444-448, 1975.

[16] A. W. T. Konings, C. T. Smit Sibinga, M. W. Aarnoudse, S. S. de Wit, and H. B. Lamberts, "Initial events in radiation-induced atheromatosis. II. Damage to intimal cells," Strahlentherapie, vol. 154, no. 11, pp. 795-800, 1978.

[17] F. Paris, Z. Fuks, A. Kang et al., "Endothelial apoptosis as the primary lesion initiating intestinal radiation damage in mice," Science, vol. 293, no. 5528, pp. 293-297, 2001.

[18] G. D. Amromin, H. L. Gildenhorn, R. D. Solomon, and B. B. Nadkarni, "The synergism of X-irradiation and cholesterolfat feeding on the development of coronary artery lesions," Journal of Atherosclerosis Research, vol. 4, pp. 325-334, 1964.

[19] R. Ross, "Cell biology of atherosclerosis," Annual Review of Physiology, vol. 57, pp. 791-804, 1995. 
[20] G. K. Hansson, "Inflammation, atherosclerosis, and coronary artery disease," The New England Journal of Medicine, vol. 352, no. 16, pp. 1685-1626, 2005.

[21] F. A. Stewart, S. Heeneman, J. Te Poele et al., "Ionizing radiation accelerates the development of atherosclerotic lesions in ApoE mice and predisposes to an inflammatory plaque phenotype prone to hemorrhage," American Journal of Pathology, vol. 168, no. 2, pp. 649-658, 2006.

[22] D. L. Tribble, M. H. Barcellos-Hoff, B. M. Chu, and E. L. Gong, "Ionizing radiation accelerates aortic lesion formation in fat-fed mice via SOD-inhibitable processes," Arteriosclerosis, Thrombosis, and Vascular Biology, vol. 19, no. 6, pp. 13871392, 1999.

[23] O. A. Hatoum, M. F. Otterson, D. Kopelman et al., "Radiation induces endothelial dysfunction in murine intestinal arterioles via enhanced production of reactive oxygen species," Arteriosclerosis, Thrombosis, and Vascular Biology, vol. 26, no. 2, pp. 287-294, 2006.

[24] N. L. Weintraub, W. K. Jones, and D. Manka, "Understanding radiation-induced vascular disease," Journal of the American College of Cardiology, vol. 55, no. 12, pp. 1237-1239, 2010.

[25] S. R. Basavaraju and C. E. Easterly, "Pathophysiological effects of radiation on atherosclerosis development and progression, and the incidence of cardiovascular complications," Medical Physics, vol. 29, no. 10, pp. 2391-2403, 2002.

[26] T. Hayashi, Y. Kusunoki, M. Hakoda et al., "Radiation dosedependent increases in inflammatory response markers in Abomb survivors," International Journal of Radiation Biology, vol. 79, no. 2, pp. 129-136, 2003.

[27] T. Hayashi, Y. Morishita, Y. Kubo et al., "Long-term effects of radiation dose on inflammatory markers in atomic bomb survivors," American Journal of Medicine, vol. 118, no. 1, pp. 83-86, 2005.

[28] M. P. Little, A. Gola, and I. Tzoulaki, "A model of cardiovascular disease giving a plausible mechanism for the effect of fractionated low-dose ionizing radiation exposure," PLoS Computational Biology, vol. 5, no. 10, Article ID e1000539, 2009.

[29] M. Boerma, C. Zurcher, I. Esveldt, C. I. Schutte-Bart, and J. Wondergem, "Histopathology of ventricles, coronary arteries and mast cell accumulation in transverse and longitudinal sections of the rat heart after irradiation," Oncology Reports, vol. 12, no. 2, pp. 213-219, 2004.

[30] Q. Zhou, Y. Zhao, P. Li, X. Bai, and C. Ruan, "Thrombomodulin as a marker of radiation-induced endothelial cell injury," Radiation Research, vol. 131, no. 3, pp. 285-289, 1992.

[31] M. M. Dunn, E. A. Drab, and D. B. Rubin, "Effects of irradiation on endothelial cell-polymorphonuclear leukocyte interactions," Journal of Applied Physiology, vol. 60, no. 6, pp. 1932-1937, 1986.

[32] J. A. Beckman, A. Thakore, B. H. Kalinowski, J. R. Harris, and M. A. Creager, "Radiation therapy impairs endotheliumdependent vasodilation in humans," Journal of the American College of Cardiology, vol. 37, no. 3, pp. 761-765, 2001.

[33] T. Sugihara, Y. Hattori, Y. Yamamoto et al., "Preferential impairment of nitric oxide-mediated endothelium-dependent relaxation in human cervical arteries after irradiation," Circulation, vol. 100, no. 6, pp. 635-641, 1999.

[34] F. Orzan, A. Brusca, M. R. Conte, P. Presbitero, and M. C. Figliomeni, "Severe coronary artery disease after radiation therapy of the chest and mediastinum: clinical presentation and treatment," British Heart Journal, vol. 69, no. 6, pp. 496$500,1993$.
[35] R. Virmani, A. Farb, A. J. Carter, and R. M. Jones, "Pathology of radiation-induced coronary artery disease in human and pig," Cardiovascular Radiation Medicine, vol. 1, no. 1, pp. 98101, 1999.

[36] R. L. Summers, "Prevalence of atypical chest pain descriptions in a population from the southern United States," American Journal of the Medical Sciences, vol. 318, no. 3, pp. 142-145, 1999.

[37] S. E. Lipshultz and S. E. Sallan, "Cardiovascular abnormalities in long-term survivors of childhood malignancy," Journal of Clinical Oncology, vol. 11, no. 7, pp. 1199-1203, 1993.

[38] K. P. Letsas, P. Korantzopoulos, D. Evangelou, L. K. Pappas, and F. Kardaras, "Acute myocardial infarction with normal coronary arteries in a patient with Hodgkin's disease: a late complication of irradiation and chemotherapy," Texas Heart Institute Journal, vol. 33, no. 4, pp. 512-514, 2006.

[39] P. H. Hardenbergh, M. T. Munley, G. C. Bentel et al., "Cardiac perfusion changes in patients treated for breast cancer with radiation therapy and doxorubicin: preliminary results," International Journal of Radiation Oncology Biology Physics, vol. 49, no. 4, pp. 1023-1028, 2001.

[40] P. Hardenbergh, M. Munley, C. Hu et al., "Doxorubicinbased chemotherapy and radiation increase cardiac perfusion changes in patients treated for left-sided breast cancer," Breast Cancer Research and Treatment, vol. 69, no. 3, p. 231, 2001.

[41] L. B. Marks, X. Yu, R. G. Prosnitz et al., "The incidence and functional consequences of RT-associated cardiac perfusion defects," International Journal of Radiation Oncology Biology Physics, vol. 63, no. 1, pp. 214-223, 2005.

[42] X. Yu, R. R. Prosnitz, S. Zhou et al., "Symptomatic cardiac events following radiation therapy for left-sided breast cancer: possible association with radiation therapy-induced changes in regional perfusion," Clinical Breast Cancer, vol. 4, no. 3, pp. 193-197, 2003.

[43] I. W. Gayed, H. H. Liu, S. W. Yusuf et al., "The prevalence of myocardial ischemia after concurrent chemoradiation therapy as detected by gated myocardial perfusion imaging in patients with esophageal cancer," Journal of Nuclear Medicine, vol. 47, no. 11, pp. 1756-1762, 2006.

[44] I. Gayed, S. Gohar, Z. Liao, M. McAleer, R. Bassett, and S. W. Yusuf, "The clinical implications of myocardial perfusion abnormalities in patients with esophageal or lung cancer after chemoradiation therapy," International Journal of Cardiovascular Imaging, vol. 25, no. 5, pp. 487-495, 2009.

[45] J. L. Anderson, C. D. Adams, E. M. Antman et al., "ACC/AHA 2007 guidelines for the management of patients with unstable angina/non ST-elevation myocardial infarction: a report of the American College of Cardiology/American Heart Association Task Force on Practice Guidelines (Writing Committee to Revise the 2002 Guidelines for the Management of Patients with Unstable Angina/Non ST-Elevation Myocardial Infarction): developed in collaboration with the American College of Emergency Physicians, the Society for Cardiovascular Angiography and Interventions, and the Society of Thoracic Surgeons: endorsed by the American Association of Cardiovascular and Pulmonary Rehabilitation and the Society for Academic Emergency Medicine," Circulation, vol. 116, no. 7, pp. e148-e304, 2007.

[46] F. Bilora, F. Pietrogrande, F. Petrobelli, G. Polato, F. Pomerri, and P. C. Muzzio, "Is radiation a risk factor for atherosclerosis? An echo-color Doppler study on Hodgkin and non-Hodgkin patients," Tumori, vol. 92, no. 4, pp. 295-298, 2006. 
[47] F. Bilora, F. Pietrogrande, E. Campagnolo et al., "Are Hodgkin and non-Hodgkin patients at a greater risk of atherosclerosis? A follow-up of 3 years," European Journal of Cancer Care, vol. 19, no. 3, pp. 417-419, 2010.

[48] S. W. K. Cheng, A. C. W. Ting, L. K. Lam, and W. I. Wei, "Carotid stenosis after radio therapy for nasopharyngeal carcinoma," Archives of Otolaryngology-Head and Neck Surgery, vol. 126, no. 4, pp. 517-521, 2000.

[49] W. W. Lam, S. F. Leung, N. M. So et al., "Incidence of carotid stenosis in nasopharyngeal carcinoma patients after radiotherapy," Cancer, vol. 92, no. 9, pp. 2357-2363, 2001.

[50] H. P. Rodemann, "Cellular basis of radiation-induced fibrosis," Radiotherapy and Oncology, vol. 35, no. 2, pp. 83-90, 1995.

[51] J. R. Stewart and L. F. Fajardo, "Radiation-induced heart disease: an update," Progress in Cardiovascular Diseases, vol. 27, no. 3, pp. 173-194, 1984.

[52] D. L. Morton, D. L. Glancy, W. L. Joseph, and P. C. Adkins, "Management of patients with radiation induced pericarditis with effusion: a note on the development of aortic regurgitation in two of them," Chest, vol. 64, no. 3, pp. 291297, 1973.

[53] M. M. Applefeld, J. F. Cole, and S. H. Pollock, "The late appearance of chronic pericardial disease in patients treated by radiotherapy for Hodgkin's disease," Annals of Internal Medicine, vol. 94, no. 3, pp. 338-341, 1981.

[54] L. H. Ling, J. K. Oh, H. V. Schaff et al., "Constrictive pericarditis in the modern era: evolving clinical spectrum and impact on outcome after pericardiectomy," Circulation, vol. 100, no. 13, pp. 1380-1386, 1999.

[55] D. A. Mulrooney, M. W. Yeazel, T. Kawashima et al., "Cardiac outcomes in a cohort of adult survivors of childhood and adolescent cancer: retrospective analysis of the Childhood Cancer Survivor Study cohort,” BMJ, vol. 339, p. b4606, 2009.

[56] K. A. Tolba and E. N. Deliargyris, "Cardiotoxicity of cancer therapy," Cancer Investigation, vol. 17, no. 6, pp. 408-422, 1999.

[57] M. Chello, P. Mastroroberto, R. Romano, S. Zofrea, I. Bevacqua, and A. R. Marchese, "Changes in the proportion of types I and III collagen in the left ventricular wall of patients with post-irradiative pericarditis," Cardiovascular Surgery, vol. 4, no. 2, pp. 222-226, 1996.

[58] R. J. Burns, B. Z. Bar Shlomo, and M. N. Druck, "Detection of radiation cardiomyopathy by gated radionuclide angiography," American Journal of Medicine, vol. 74, no. 2, pp. 297-302, 1983.

[59] L. S. Constine, R. G. Schwartz, D. E. Savage, V. King, and A. Muhs, "Cardiac function, perfusion, and morbidity in irradiated long-term survivors of Hodgkin's disease," International Journal of Radiation Oncology Biology Physics, vol. 39, no. 4, pp. 897-906, 1997.

[60] G. Gyenes, T. Fornander, P. Carlens, U. Glas, and L. E. Rutqvist, "Myocardial damage in breast cancer patients treated with adjuvant radiotherapy: a prospective study," International Journal of Radiation Oncology Biology Physics, vol. 36, no. 4, pp. 899-905, 1996.

[61] M. J. Adams, P. H. Hardenbergh, L. S. Constine, and S. E. Lipshultz, "Radiation-associated cardiovascular disease," Critical Reviews in Oncology/Hematology, vol. 45, no. 1, pp. 55-75, 2003.

[62] S. A. Hunt, W. T. Abraham, M. H. Chin et al., "2009 Focused update incorporated into the ACC/AHA 2005 Guidelines for the Diagnosis and Management of Heart Failure in Adults. A Report of the American College of Cardiology Foundation/American Heart Association Task Force on Practice
Guidelines Developed in Collaboration with the International Society for Heart and Lung Transplantation," Journal of the American College of Cardiology, vol. 53, no. 15, pp. e1-e90, 2009.

[63] R. Yarom, I. S. Harper, S. Wynchank et al., "Effect of captopril on changes in rats' hearts induced by long-term irradiation," Radiation Research, vol. 133, no. 2, pp. 187-197, 1993.

[64] A. Tamura, Y. Takahara, K. Mogi, and M. Katsumata, "Radiation-induced valvular disease is the logical consequence of irradiation," General Thoracic and Cardiovascular Surgery, vol. 55, no. 2, pp. 53-56, 2007.

[65] R. G. Carlson, W. R. Mayfield, S. Normann, and J. A. Alexander, "Radiation-associated valvular disease," Chest, vol. 99, no. 3, pp. 538-545, 1991.

[66] M. D. Brand, C. A. Abadi, G. P. Aurigemma, H. L. Dauerman, and T. E. Meyer, "Radiation-associated valvular heart disease in Hodgkin's disease is associated with characteristic thickening and fibrosis of the aortic-mitral curtain," Journal of Heart Valve Disease, vol. 10, no. 5, pp. 681-685, 2001.

[67] N. M. Katz, A. W. Hall, and M. D. Cerqueira, "Radiation induced valvulitis with late leaflet rupture," Heart, vol. 86, no. 6, p. E20, 2001.

[68] K. J. Totterman, E. Pesonen, and P. Siltanen, "Radiationrelated chronic heart disease," Chest, vol. 83, no. 6, pp. 875878, 1983.

[69] M. S. Slama, D. Le Guludec, C. Sebag et al., "Complete atrioventricular block following mediastinal irradiation: a report of six cases," Pacing and Clinical Electrophysiology, vol. 14, no. 7, pp. 1112-1118, 1991.

[70] L. La Vecchia, "Physiologic dual chamber pacing in radiationinduced atrioventricular block," Chest, vol. 110, no. 2, pp. 580$581,1996$.

[71] S. I. Cohen, S. Bharati, J. Glass, and M. Lev, "Radiotherapy as a cause of complete atrioventricular block in Hodgkin's disease. An electrophysiological-pathological correlation," Archives of Internal Medicine, vol. 141, no. 5, pp. 676-679, 1981.

[72] T. Nakao, H. Kanaya, M. Namura et al., "Complete atrioventricular block following radiation therapy for malignant thymoma," Japanese Journal of Medicine, vol. 29, no. 1, pp. 104-110, 1990.

[73] L. Hughes-Davies, D. B. Sacks, J. Rescigno, S. Howard, and J. Harris, "Serum cardiac troponin T levels during treatment of early-stage breast cancer," Journal of Clinical Oncology, vol. 13, no. 10, pp. 2582-2584, 1995.

[74] K. R. Kozak, T. S. Hong, P. M. Sluss et al., "Cardiac blood biomarkers in patients receiving thoracic (chemo)radiation," Lung Cancer, vol. 62, no. 3, pp. 351-355, 2008.

[75] U. Nellessen, M. Zingel, H. Hecker, J. Bahnsen, and D. Borschke, "Effects of radiation therapy on myocardial cell integrity and pump function: which role for cardiac biomarkers?" Chemotherapy, vol. 56, no. 2, pp. 147-152, 2010.

[76] J. Rademaker, H. Schöder, N. S. Ariaratnam et al., "Coronary artery disease after radiation therapy for Hodgkin's lymphoma: coronary CT angiography findings and calcium scores in nine asymptomatic patients," American Journal of Roentgenology, vol. 191, no. 1, pp. 32-37, 2008. 


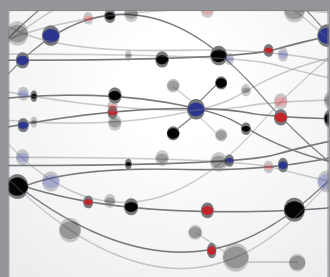

The Scientific World Journal
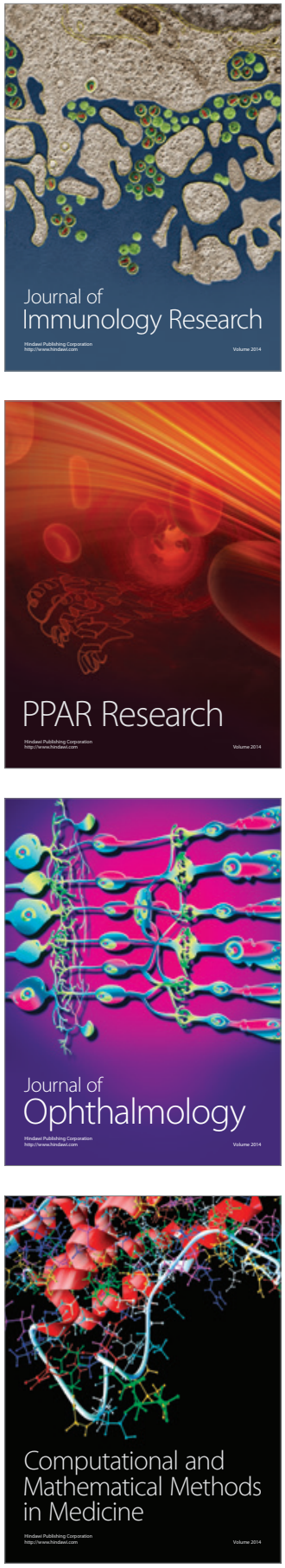

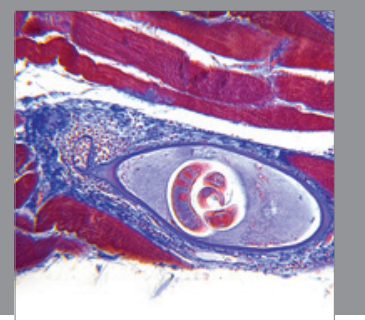

Gastroenterology

Research and Practice
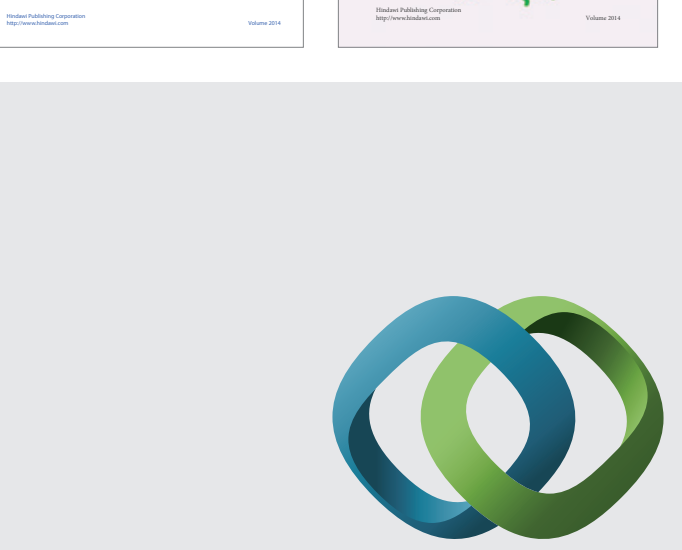

\section{Hindawi}

Submit your manuscripts at

http://www.hindawi.com
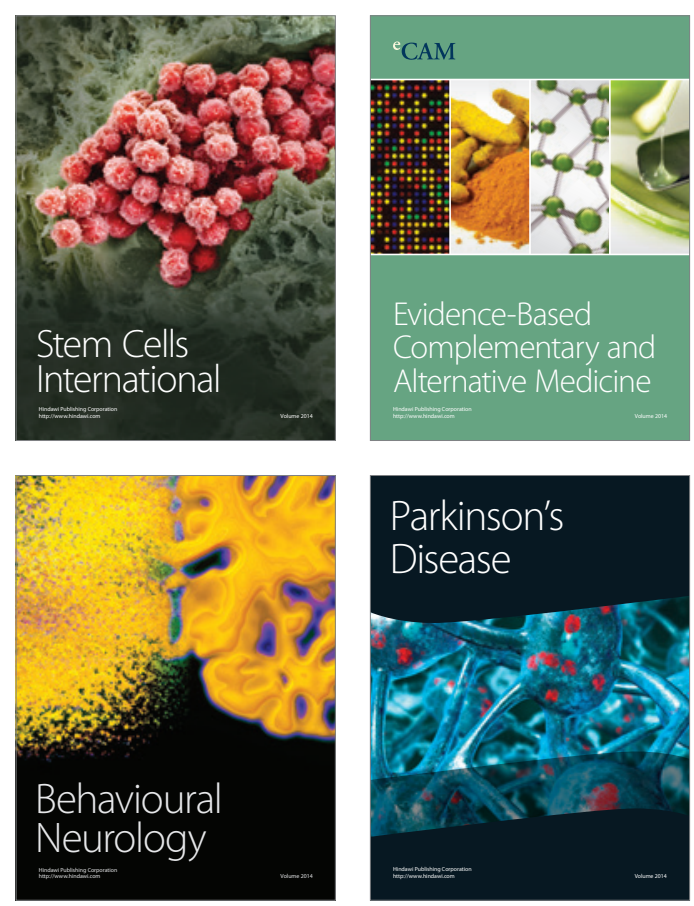

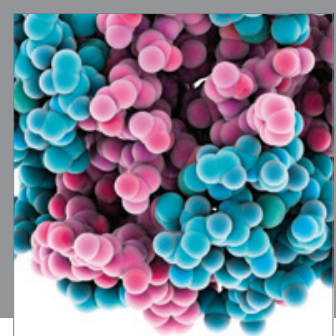

Journal of
Diabetes Research

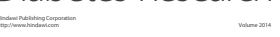

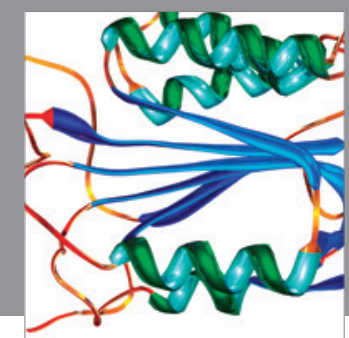

Disease Markers
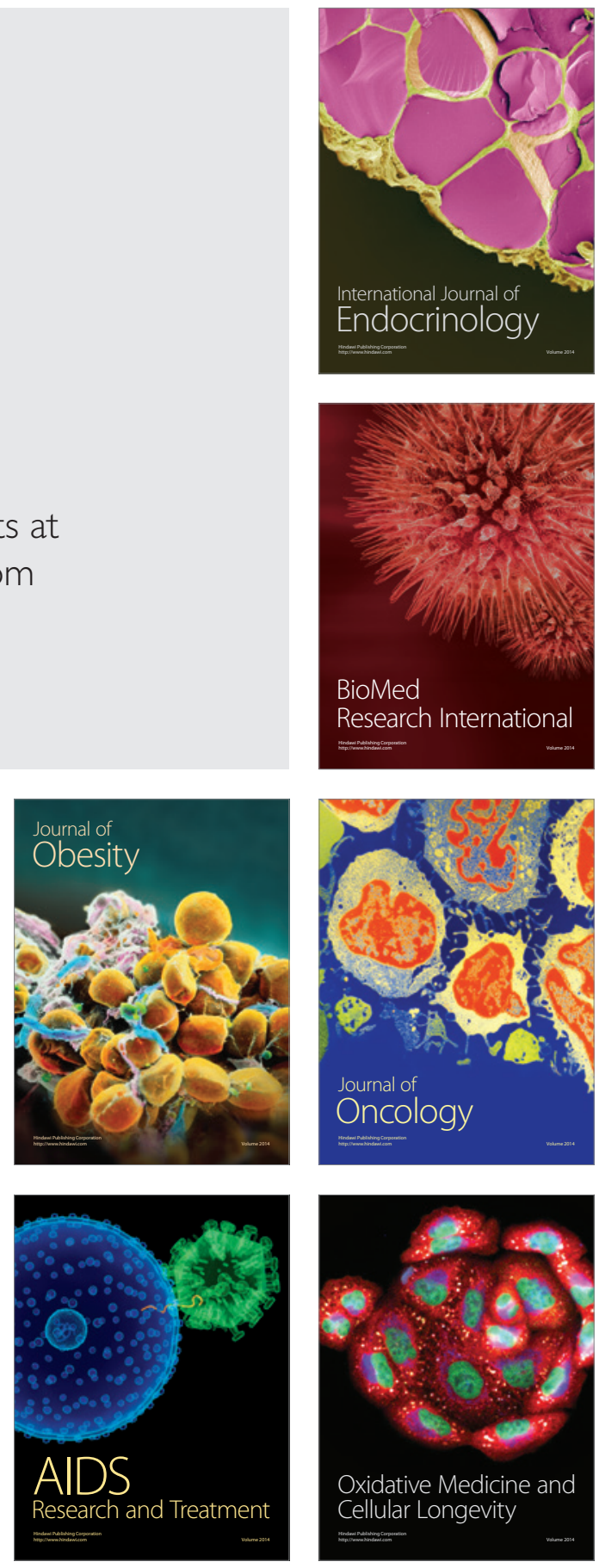